\section{CVIA}

CASE REPORT

pISSN 2508-707X / eISSN 2508-7088 https://doi.org/10.22468/cvia.2017.00122 CVIA 2017;1(4):251-254

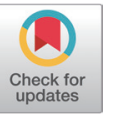

\title{
Demonstration of Turbulence at the Site of Ascending Aorta Graft Kinking Using 4-Dimensional Flow Magnetic Resonance Imaging
}

\author{
Hyun Jung Koo', Joon-Won Kang', Hojin $\mathrm{Ha}^{2}$, \\ Dong Hyun Yang', Suk-Jung Choo ${ }^{3}$, Cheol Hyun Chung', \\ Jae-Kwan Song ${ }^{4}$, Tae-Hwan Lim ${ }^{1}$ \\ 'Department of Radiology and Research Institute of Radiology, \\ ${ }^{3}$ Department of Cardiovascular Surgery, ${ }^{4}$ Division of Cardiology, Cardiac Imaging Center, \\ Asan Medical Center, University of Ulsan College of Medicine, Seoul, Korea \\ ${ }^{2}$ Department of Mechanical and Biomedical Engineering, Kangwon National University, \\ Chuncheon, Korea
}

\begin{abstract}
A 52-year-old female with ascending aorta graft kinking with a protruding inward flap after ascending aorta and total arch replacement was assessed with four-dimensional (4D) flow magnetic resonance imaging (MRI) on a 3.0 Tesla MR scanner (MAGNETOM Skyra; Siemens, Munich, Germany). The 4D flow direction, velocity, and shear stress analyses demonstrated turbulent flow with increased flow acceleration and wall shear stress at the kinking site of the ascending aorta. After surgical graft revision, 4D MRI showed decreased turbulence and resolution of the abnormally increased velocity and shear stress at the site.
\end{abstract}

Key words Aorta $4 \mathrm{D}$ flow · Magnetic resonance imaging.

\section{INTRODUCTION}

Recently, time-resolved, flow-sensitive, magnetic resonance imaging (MRI), namely four-dimensional (4D) flow MRI, has been used in assessing many cardiovascular diseases, including aortic disease. The measurement of blood flow velocity and wall shear stress by this MRI technique helps reveal flow dynamics, and its utility in evaluating the potential risks of altered aortic hemodynamics has been investigated in several studies [1-3].

\section{CASE REPORT}

A 52-year-old woman who had undergone ascending aorta and total arch replacement with proximal descending aortic stent grafting at an outside hospital for aortic dissection three years earlier presented to our outpatient clinic with recurrent hemolytic anemia. Two graft techniques involving a separate ascending aortic tube graft and a four-branch graft for arch re-

(c) This is an Open Access article distributed under the terms of the Creative Commons Attribution Non-Commercial License (http://creativecommons.org/licenses/by$\mathrm{nc} / 4.0$ ) which permits unrestricted non-commercial use, distribution, and reproduction in any medium, provided the original work is properly cited. placement was performed. The two graft segments that were sutured together met at the mid-ascending aorta level. The descending thoracic aortic stent graft was implanted during follow-up, presumably to induce false lumen remodeling. After the aortic operation, the patient received 2-3 packs of red blood cells (RBCs) once every two weeks when she suffered severe dizziness and syncope. Her RBC count was $2.4 \times 10^{6} \mu \mathrm{L}$ (normal range: $4.0 \times 10^{6}-5.4 \times 10^{6} \mu \mathrm{L}$ ) with increased level of reticulocytes (11.2\%, normal range: $0.5-1.8 \%)$. The patient's hemoglobin was $8.1 \mathrm{~g} / \mathrm{dL}$ (normal range: $12-16 \mathrm{~g} / \mathrm{dL}$ ), and she had a low level of haptoglobin, below $7.8 \mathrm{mg} / \mathrm{dL}$ (normal range: 30-200 mg/ dL). A peripheral blood smear showed fragmented RBCs. She had no history of hematologic malignancy, and her spleen was normal size. Transthoracic echocardiography revealed abnormal linear echogenic material causing significant obstruction at the level of the proximal aortic graft with jet flow in the distal part of the ascending aorta. On transesophageal echocardiography (TEE), a linear echogenic structure was found protruding into the aortic lumen at the mid-ascending aortic graft level, causing severe stenosis (Supplementary Movie 1 in the onlineonly Data Supplement, Fig. 1A). The maximal velocity in the 
stenotic area was $5.1 \mathrm{~m} / \mathrm{s}$, with a pressure gradient of $106 / 58 \mathrm{~mm}$ Hg (Fig. 1B).

An electrocardiographically-gated, sagittal, computed tomography (CT) image showed kinking of the ascending portion of the aortic graft with angulation resulting in an internally protruding $0.8 \mathrm{~cm}$ to $1 \mathrm{~cm}$ linear structure (Fig. 2A). There was no evidence of vegetation or abscess formation. The diameter of the ascending aorta (graft) was $2.9 \mathrm{~cm}$, but the luminal diameter at the level of the protrusion was $1.4 \mathrm{~cm}$. To evaluate potential altered hemodynamics, including turbulent flow, at the kinked aorta, the patient underwent 4D flow MRI. On MRI, severe flow acceleration with increased wall shear stress was noted at the kinking site of the ascending aorta (Fig. 3).

Aortic graft revision was planned to remove the protruding obstructive structure. In the surgical field, a $2 \mathrm{~cm}$ inward flap was noted in the ascending aorta, 5-7 cm distal to the aortic root. The size and shape of the flap correlated well with those identified on echocardiography and CT. The flap was caused by infolding of an overly redundant ascending aortic portion of the four-branch graft. After surgical revision, the patient underwent $4 \mathrm{D}$ flow MRI to evaluate any changes in turbulence at the kinking site (Fig. 3). According to the $4 \mathrm{D}$ flow image, the severe flow disturbance with sudden directional changes in blood flow was relieved after surgery (Fig. 3B). The overall flow direc-
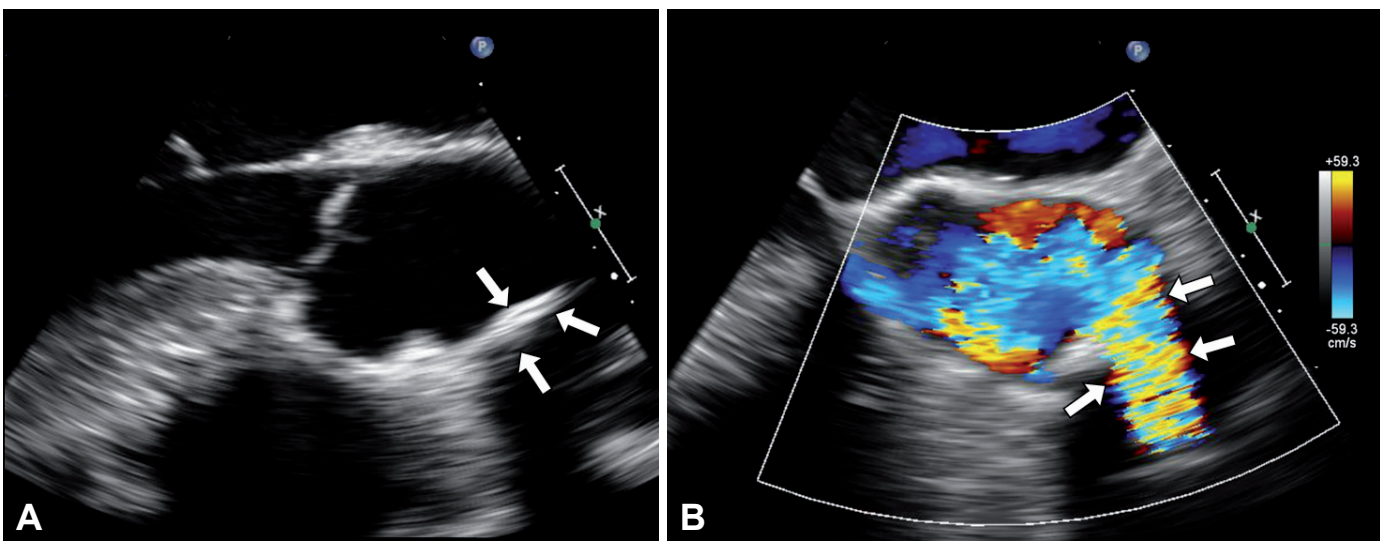

Fig. 1. TEE obtained before aortic graft revision. (A) On TEE, a linear echogenic strut (arrows) protruding into the ascending aorta lumen at the junction between the native aorta and proximal aortic graft was evident. (B) A color Doppler image showed severe stenosis with jet (arrows) at the strut level, with a maximal velocity of $5.1 \mathrm{~m} / \mathrm{s}$. TEE: transesophageal echocardiography.
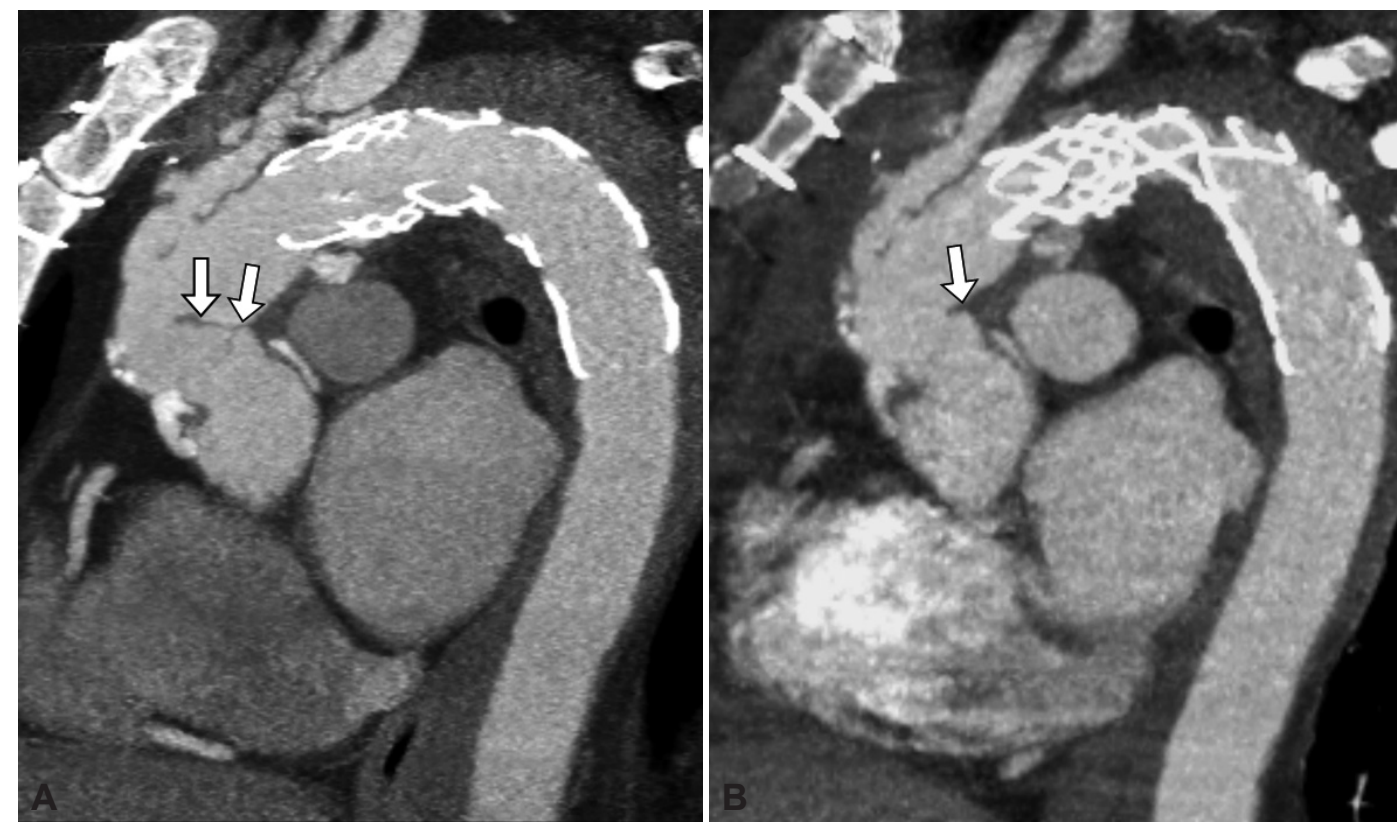

Fig. 2. Sagittal images of reconstructed CT images. (A) A preoperative CT image showing a $1.8 \mathrm{~cm}$ linear structure within the aorta, located at the junction of the angulated proximal ascending aorta above the aortic root. An aortic arch stent graft was in place. (B) After revision of the aortic graft with removal of the strut, a postoperative CT image showed a shortened strut (arrow) compared to the preoperative CT image. CT: computed tomography. 

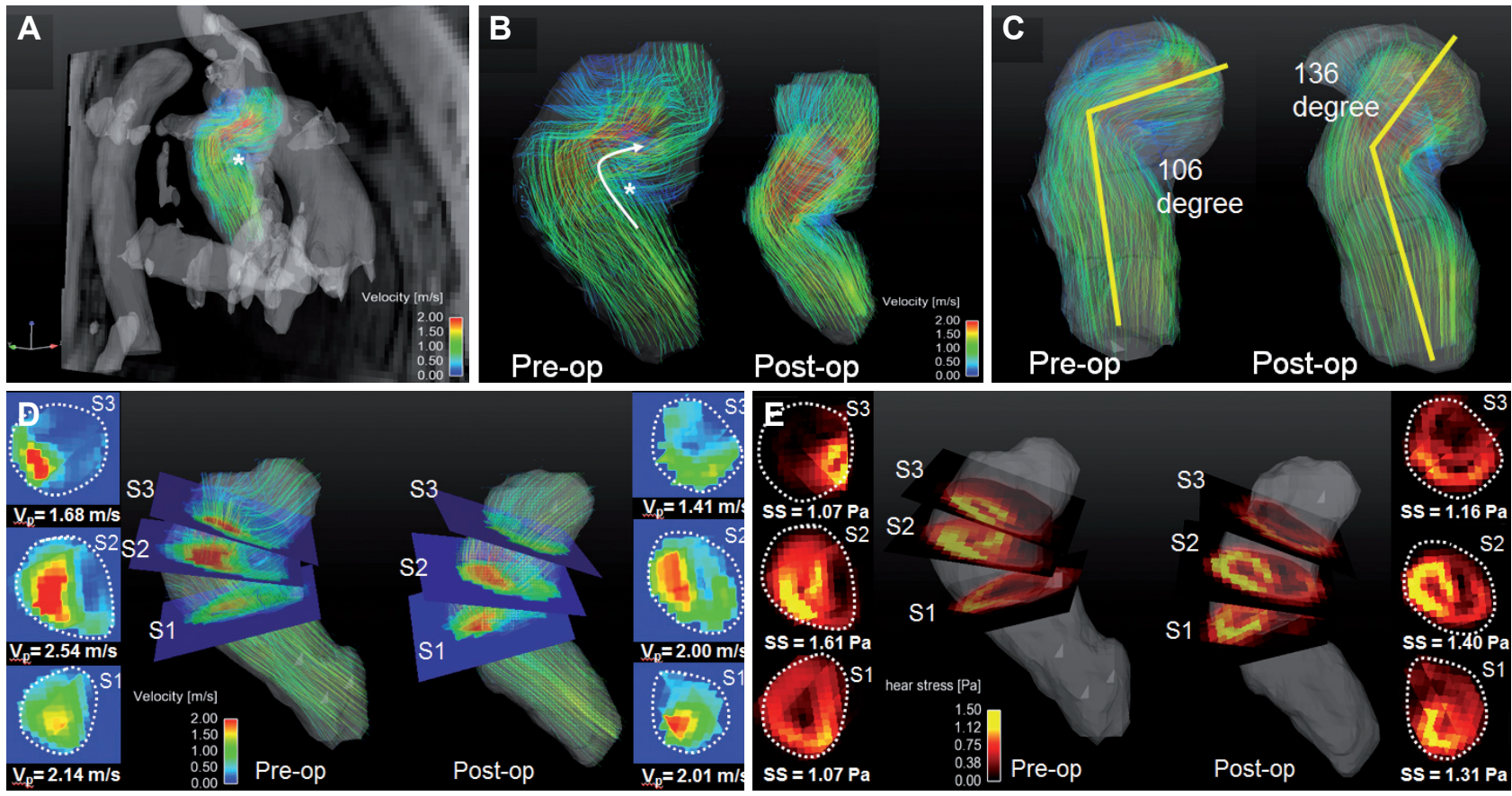

Fig. 3. Comparison of 4D flow MR images of the ascending aorta blood flow before and after surgery. (A) Preoperative 4D flow MR image showing that the graft strut (asterisk) disturbed the direction of the aortic blood flow. (B) Comparison of velocity profiles at the kinked ascending aorta between preoperative and postoperative 4D flow MR images. Note that the severe flow disturbance with sudden directional changes in blood flow was relieved after the operation. (C and D) Comparison of cross-sectional velocity distributions at three slices (S1, S2, and S3 indicate the three sections) at the ascending aorta. The peak velocities at the region near the stenosis (S2 and S3) were reduced after the operation. (E) Cross-sectional shear stress profiles of the kinked aorta at each point. The cardiac phases of the velocity field and shear stress profiles in the figures were obtained at the peak systolic phase. 4D: four-dimensional.

tion was changed from $106^{\circ}$ to $136^{\circ}$ (Fig. 3C). The maximal velocity at the kinking site was decreased from $2.5 \mathrm{~m} / \mathrm{s}$ to $2.0 \mathrm{~m} / \mathrm{s}$, and the maximal shear stress at the site was decreased from 1.61 $\mathrm{Pa}$ to $1.40 \mathrm{~Pa}$ (Fig. 3D and E). Postoperative TEE showed relieved obstruction of the ascending aorta graft anastomosis site (Supplementary Movie 2 in the online-only Data Supplement), and CT demonstrated resolving stenosis at the revision site of the ascending aorta (Fig. 2B). Although residual flow acceleration at the level of the graft infolding persisted, the maximal velocity decreased to $3.4 \mathrm{~m} / \mathrm{s}$, and the pressure gradient was $57 / 28$ $\mathrm{mm} \mathrm{Hg}$. One month after the operation, the anemia had improved, and the patient had a normal RBC count $\left(4.5 \times 10^{6} \mu \mathrm{L}\right)$ and normal hemoglobin $(13.4 \mathrm{~g} / \mathrm{dL})$ level. The patient's symptoms, including dizziness and syncope, had also improved significantly, along with resolution of dark urinary discoloration.

\section{DISCUSSION}

Recent studies have shown that 4D flow-sensitive MRI can be used to demonstrate blood flow in a thoracic aortic dissection or aneurysm $[1,2]$, even when complex anatomic anomalies, such as an interrupted aortic arch, are involved [3]. Turbulence or helical flow patterns in the diseased aorta can be clues for the need for a therapeutic plan. The reproducibility of aor- tic blood flow analysis using 4D flow MRI is good, showing moderate agreement with wall shear stress [4]. The functional problems of aortic diseases might not be completely evaluated using echocardiography alone, because of the limited sonic window. Complete views cannot be obtained in a one-shot image, not only for deep-seated lesions in the aortic arch or descending aorta, but also for the ascending aorta, and the sonic window can be interrupted by obstacles such as the sternum or ribs. Although TEE can overcome these obstacles, $4 \mathrm{D}$ flow dynamics might not be properly generated using echocardiography alone. For evaluating the hemodynamic significance of abnormal lesions that can cause blood flow disturbance, $4 \mathrm{D}$ flow MRI can be used to identify whether the lesion truly affects flow changes. Postoperative MR images can be used to evaluate the improved state of stenosis through a comparison with preoperative images. Our current case demonstrated that aorta 4D flow MRI was useful for providing hemodynamic information on a lesion that correlated well with the clinical findings. Hence, 4D flow MRI can provide qualitative and quantitative information for the evaluation of blood flow kinetics at the ascending aorta in order to demonstrate hemodynamically significant stenosis.

\section{Supplementary Movie Legends}

Movie 1. Transesophageal echocardiography cines demonstrating severe 
stenosis at the level of the linear strut between the native aorta and proximal aortic graft. The maximal velocity was $5.1 \mathrm{~m} / \mathrm{s}$.

Movie 2. Postoperative transesophageal echocardiography cines showing improved stenosis at the ascending aorta with decreased flow acceleration at the level of the strut. The maximal velocity decreased to $3.4 \mathrm{~m} / \mathrm{s}$, and the pressure gradient was $57 / 28 \mathrm{~mm} \mathrm{Hg}$.

\section{Supplementary Materials}

The online-only Data Supplement is available with this article at https:// doi.org/10.22468/cvia.2017.00122.

\section{Conflicts of Interest}

The authors declare that they have no conflict of interest.

\section{REFERENCES}

1. François CJ, Markl M, Schiebler ML, Niespodzany E, Landgraf BR,
Schlensak C, et al. Four-dimensional, flow-sensitive magnetic resonance imaging of blood flow patterns in thoracic aortic dissections. J Thorac Cardiovasc Surg 2013;145:1359-1366.

2. Hope TA, Markl M, Wigström L, Alley MT, Miller DC, Herfkens RJ. Comparison of flow patterns in ascending aortic aneurysms and volunteers using four-dimensional magnetic resonance velocity mapping. J Magn Reson Imaging 2007;26:1471-1479.

3. Hirtler D, Geiger J, Jung B, Markl M, Arnold R. 4-D MRI flow analysis in the course of interrupted aortic arch reveals complex morphology and quantifies amount of collateral blood flow. Pediatr Radiol 2013;43:10371040 .

4. Markl M, Wallis W, Harloff A. Reproducibility of flow and wall shear stress analysis using flow-sensitive four-dimensional MRI. J Magn Reson Imaging 2011;33:988-994. 